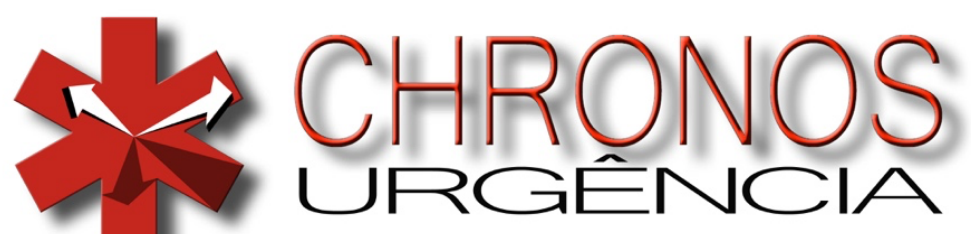

https://chronos.samu.fortaleza.ce.gov.br eISSN 2763-5872

\title{
Artigos
}

\section{Caracterização dos Ensaios Clínicos Randomizados sobre Urgência e Emergência Realizados por Enfer- meiros no Brasil}

\author{
Characterization of Randomized Clinical Trials on Urgency and \\ Emergency Carried out by Nurses in Brazil (abstract: p. 10)
}

\section{Caracterización de Ensayos Clínicos de Urgencia y Emergencia Alea- torizados Realizados por Enfermeras en Brasil (resumen: p. 10)}

\author{
(iD) ANA CLARA ALVES DE BRITO \\ acab@discente.ifpe.edu.br \\ IFPE Campus Pesqueira \\ BR 232, Km 208, s/n. Bairro: Prado. Pesqueira-PE. CEP: 55200-000

\section{RAQUEL GOUVEIA RAMOS} \\ raquelgr8@hotmail.com \\ IFPE Campus Pesqueira \\ (iD) GIRLEIDE ISÍDIO DA SILVA \\ giirleideisidio@gmail.com \\ IFPE Campus Pesqueira
}

\section{(iD) ANA KAROLINA FÉLIX DE ALMEIDA}

karolinaanakarolina28@gmail.com

IFPE Campus Pesqueira

(iD) GUILHERME GUARINO DE MOURA SÁ

guilherme_mourasa@hotmail.com

IFPE Campus Belo Jardim

NELSON MIGUEL GALINDO NETO

nelsongalindont@hotmail.com

IFPE Campus Pesqueira

Objetivo: Realizar a caracterização dos ensaios clínicos randomizados sobre urgência e emergência realizados por enfermeiros no Brasil. Método: Estudo descritivo, quantitativo. Realizado em laboratório do Instituto Federal de Pernambuco Campus Pesqueira, por intermédio de acesso ao banco de dados online do Registro Brasileiro de Ensaios Clínicos. Na coleta dos dados aplicou-se instrumento, composto por 23 questões que foram preenchidas de acordo com informações de cada estudo. Para análise dos dados foi utilizado o software Microsoft Excel 2016. Resultados: Dos 321 estudos cadastrados na ReBEC, sete $(2,1 \%)$ correspondiam a pesquisas realizadas sobre urgência e emergência. Quanto ao público avaliado, dois estudos (28,6\%) investigaram enfermeiros, dois $(28,6 \%)$ foram realizados com estudantes de enfermagem, dois (28,6\%) pesquisaram pacientes e um $(14,2 \%)$ foi constituído para estudantes surdos. No tocante ao tipo de mascaramento, cinco $(71,4 \%)$ estudos foram abertos; um $(14,3 \%)$ do tipo unicego e um $(14,3 \%)$ duplo-cego. Todos os autores encontravam-se filiados a instituições públicas de caráter Federal ou Estadual. Conclusão: Este estudo aponta a limitada atuação da enfermagem na realização de Ensaios Clínicos Randomizados relacionados a urgência e emergência no Brasil.

Descritores: Ensaios Clínicos Randomizados; Enfermagem; Urgência.

Palavras-chave: Enfermeiros; Ensaios clínicos; Urgência e Emergência. 


\section{Introdução}

Sabe-se que os ensaios clínicos randomizados são estudos experimentais amplamente realizados com seres humanos, nos quais os participantes são sorteados de modo aleatório para participar da aplicação de alguma intervenção ou teste experimental, logo, neste tipo de estudo, um grupo de pessoas é exposto a determinado tipo de intervenção, onde é comparado com um grupo denominado controle. Os ensaios clínicos randomizados que são bem conduzidos tornam-se modelos, no que concerne ao alcance de resultados positivos, o que contribui e direciona as práticas clínicas cotidianas ${ }^{1}$.

A finalidade de um Ensaio Clínico Randomizado (ECR) é determinar a efetividade e seguridade de intervenções realizadas no âmbito da saúde, uma vez que seus achados possibilitam o estabelecimento de causa e efeito. Assim, o ECR aponta intervenções que podem ser aplicadas na prática clínica de forma que pode contribuir para a melhora da saúde².

A execução de ensaios clínicos randomizados por enfermeiros é relevante, diante da ampla atuação dessa categoria profissional e da importância da sua atuação para a prevenção, promoção, tratamento e reabilitação da saúde. Isto contribui para o desenvolvimento do cuidado seguro, cientificamente respaldado ${ }^{3}$.

A atuação dos enfermeiros nos setores de urgência e emergência pré e intra hospitalar é decisório no ato de salvar vidas. As funções da enfermagem na conjunção de urgência e emergência são vastas, dado que a assistência prestada por estes profissionais envolve o cuidado com pacientes em condições graves, além de intervenções que objetivam tratar condições agudas que oferecem riscos à $v i^{4}{ }^{4}$. A enfermagem tem se alicerçado como ciência e a atuação dos enfermeiros requer evidências científicas, de modo que se torna pertinente a realização de ECR por enfermeiros na área de urgência e emergência para respaldar e expandir o campo de prática e atuação profissional ${ }^{5}$.

É pertinente que seja traçado o perfil dos Ensaios Clínicos Randomizados já existentes e cadastrados, a fim de auxiliar, compreender o contexto e apontar lacunas a serem contempladas em futuras pesquisas científicas. Desse modo, este estudo teve como objetivo realizar a caracterização dos Ensaios Clínicos Randomizados sobre urgência e emergência realizados por enfermeiros no Brasil. 


\section{Metodologia}

Tratou-se de estudo descritivo, de natureza quantitativa, realizado em laboratório do Instituto Federal de Educação, Ciência e Tecnologia de Pernambuco Campus Pesqueira, por intermédio de acesso ao banco de dados online do Registro Brasileiro de Ensaios Clínicos.

A ReBEC refere-se a um website brasileiro de domínio público, com natureza científica, administrado pela Fundação Oswaldo Cruz, onde são registrados os Ensaios Clínicos Randomizados com seres humanos no Brasil. Trata-se de relevante fonte de pesquisas e os ECR, para serem publicados, necessitam de registro nessa plataforma online.

A amostra foi composta pelos ECR registrados no banco de dados da ReBEC. No que se refere aos critérios de inclusão da amostra, foram incluídos todos os ECR executados no Brasil que foram registrados por profissionais enfermeiros (a). A constatação do grupo profissional, se deu a partir da visitação ao currículo Lattes do autor que cadastrou o ensaio clínico na ReBEC. Como parâmetro de exclusão aplicado, foram excluídos os estudos que consistiam como cancelado/interrompido.

Na coleta dos dados aplicou-se instrumento elaborado para este estudo, composto por 23 questões que foram preenchidas de acordo com as informações específicas de cada estudo e particularidades do pesquisador/autor. As variáveis referiam-se a: tipo de estudo; público avaliado; quantos participantes; agravo/condição de saúde estudado; qual intervenção realizada; área da enfermagem; nível de assistência em saúde; setor; quantos grupos foram comparados; idade mínima e máxima para inclusão; intervalo de tempo entre as medidas pré e pós; situação do recrutamento; ano do recrutamento; tipo do mascaramento; foco do estudo; tipo de alocação; desfecho primário; filiação do autor; título do autor; atividade exercida pelo autor; estado e região da filiação.

Na ocasião do acesso virtual ao website para a coleta de dados o Registro Brasileiro de Ensaios Clínicos disponibilizava de 321 estudos registrados. Após a leitura detalhada dos títulos e resumos desses estudos, 314 foram excluídos por não discorrerem sobre Urgência e Emergência, de modo que apenas sete abordavam urgência e emergência e foram selecionados para integrarem a amostra.

Para análise dos dados foi utilizado o software Microsoft Excel 2016. No que concerne aos aspectos éticos, não foi necessário a avaliação do comitê de ética e pesquisa com seres humanos, por tratar-se de informações de domínio público. 


\section{Resultados}

Dos 321 estudos cadastrados na ReBEC, apenas sete $(2,1 \%)$ correspondiam a pesquisas realizadas com relação a urgência e emergência, das quais quatro $(57,0 \%)$ compararam dois grupos e duas $(28,6 \%)$ eram estudos quase-experimentais. Quanto ao público avaliado dois $(28,6 \%)$ pesquisaram enfermeiros; dois (28,6\%), estudantes de enfermagem; dois $(28,6 \%)$, pacientes e um $(14,2 \%)$ investigou estudantes surdos.

Acerca da amostra dos estudos, três (42,8\%) contaram com 30 participantes, cada; um $(14,3 \%)$ contou com 270 participantes, um estudo (14,3\%) com 113, um (14,3\%) obteve 94 integrantes e um $(14,3 \%)$ com 61 participantes.

Em relação ao agravo de saúde estudado e à intervenção testada, houve predominância de pesquisas sobre o uso da simulação realística para investigação da temática referente à parada cardiorrespiratória, encontradas em dois $(28,5 \%)$ estudos. Os agravos estudados e as intervenções testadas encontram-se detalhados na Tabela 1.

Tabela 1. Descrição do problema e intervenção pesquisados nos ensaios clínicos cadastrados por enfermeiros. Pesqueira, PE, Brasil, 2021.

\begin{tabular}{|c|c|}
\hline Condição de saúde estudada & $\mathrm{N}(\%)$ \\
\hline Parada cardiorrespiratória & $2(28,5)$ \\
\hline Afecções cardíacas, exame físico, cuidados de enfermagem & $1(14,3)$ \\
\hline Ferimentos e lesões, infecções, viroses, distúrbios químicos, pneumonia & $1(14,3)$ \\
\hline Nível de dor na administração intramuscular da Benzilpenicilina Benzatina & $1(14,3)$ \\
\hline Conhecimento dos profissionais, condições patológicas, sinais e sintomas & $1(14,3)$ \\
\hline Pessoas com deficiência auditiva; ressuscitação Cardiopulmonar, conhecimento e destreza motora & $1(14,3)$ \\
\hline $\begin{array}{ll}\text { Intervenção realizada } & \text { Inad }\end{array}$ & $\mathrm{N}(\%)$ \\
\hline Simulação clínica com uso de manequins de baixa e alta fidelidade. & $2(28,5)$ \\
\hline Anamnese, exame físico, administração de medicamentos, monitorização cardíaca, ressuscitação cardiopulmonar & $1(14,3)$ \\
\hline Entrevista semiestruturada e verificação de prontuários & $1(14,3)$ \\
\hline Administração de fármaco Benzilpenicilina Benzatina G (benzetacil). & $1(14,3)$ \\
\hline Educação em saúde sobre Reanimação Cardiopulmonar. & $1(14,3)$ \\
\hline Vídeo educativo sobre Ressuscitação Cardiopulmonar (RCP). Aula expositiva tradicional. & $1(14,3)$ \\
\hline
\end{tabular}

No que se refere à subárea da emergência, quatro $(57,1 \%)$ estudos correspondiam ao contexto hospitalar e três ao pré-hospitalar (42,9\%). No tocante ao setor/local de realização da pesquisa, dois estudos (28,5\%) ocorreram no setor laboratório/universidade, enquanto o desenvolvimento em UTI Neonatal, Unidade de Pediatria, Centro de Parto Normal, transferência hospitalar, pronto socorro, hospital e escola, foram encontrados em um (14,3\%) estudo, cada. 
Sobre a idade mínima e máxima para inclusão, a mínima variou de 0 a 18 anos, enquanto a idade máxima variou de 0 a 100 anos. No tangente ao intervalo de tempo entre as medidas pré e pós, predominaram dois estudos $(28,5 \%)$ que definiram intervalo de 15 dias, como detalha a Tabela 2 .

Tabela 2. Descrição de idade mínima e máxima para inclusão e intervalo de tempo entre as medidas pré e pós. Pesqueira, PE. 2021.

\begin{tabular}{rc}
\hline Idade mínima para inclusão & $\mathrm{N}(\%)$ \\
18 anos & $5(71,4)$ \\
14 anos & $1(14,3)$ \\
0 anos & $1(14,3)$ \\
\hline Idade máxima para inclusão & $\mathrm{N}(\%)$ \\
\hline 100 anos & $1(14,3)$ \\
70 anos & $1(14,3)$ \\
40 anos & $1(14,3)$ \\
0 anos & $1(14,3)$ \\
\hline Informação encontrava-se indisponível & $3(42,8)$ \\
\hline Intervalo de tempo entre as medidas pré e pós & $\mathrm{N}(\%)$ \\
15 dias & $2(28,5)$ \\
1 ano & $1(14,3)$ \\
2 meses & $1(14,3)$ \\
\hline Informação encontrava-se indisponível & $3(42,9)$ \\
\hline
\end{tabular}

No que concerne ao ano de recrutamento um estudo (14,3\%) foi realizado em 2012; um $(14,3 \%)$ em 2016; um (14,3\%) ocorreu entre os anos de 2017 e 2018; um (14,3\%) entre 2015 e 2016; um $(14,3 \%)$ no ano de 2019 e dois $(28,5 \%)$ no ano de 2017 , de forma que não se observa avanço quantitativo ao longo dos anos. No âmbito do tipo de mascaramento, cinco $(71,4 \%)$ estudos foram abertos; um (14,3\%) do tipo unicego e um $(14,3 \%)$ duplo-cego.

Sobre os desfechos primários, em um estudo (14,3\%) pesquisou se membros de uma equipe de enfermagem, que receberam capacitação e feedback sobre seu desempenho, obtiveram melhores resultados na realização do exame clínico objetivo estruturado (OSCE), quando comparados ao grupo que realizou treino autodidata e não foi exposto a feedback. Outro estudo (14,3\%) teve como desfecho primário a satisfação dos pacientes. Um $(14,3 \%)$ investigou o aumento do número de intervenções da Sistematização da Assistência de Enfermagem (SAE) inadequadas por falta de preparo/treinamento. Outro estudo $(14,3 \%)$ verificou a aceitação do uso do medicamento (benzetacil) pelos pacientes. Em dois $(28,6 \%)$ estudos foi investigada se estudantes demonstrariam maior autoconfiança e conhecimento no que concerne à ressuscitação cardiopulmonar e atuação em situações de emergência após simulação. E o último estudo (14,3\%) deteve como 
desfecho primário o conhecimento e habilidade de estudantes surdos sobre parada cardiorrespiratória.

Todos os autores encontravam-se filiados a instituições públicas de caráter Federal ou Estadual. Relativo à titulação do autor, sobressaíram-se os com mestrado, encontrados em três $(42,9 \%)$ estudos. Acerca da atuação profissional, predominaram os que atuavam na docência e pesquisa, encontrados em quatro $(57,1 \%)$ estudos, como detalha a Tabela 3.

Tabela 3. Descrição de filiação, titulação e exercício profissional do autor. Pesqueira, PE. 2021.

\begin{tabular}{rr}
\hline Instituição de Filiação do autor/ Estado & $\mathrm{N}(\%)$ \\
\hline Universidade Federal do Rio de Janeiro (UFRJ) / Rio de Janeiro & $2(28,5)$ \\
Universidade do Estado do Rio Grande do Norte, (UERN) / Rio Grande do Norte & $1(14,3)$ \\
Universidade Regional do Cariri, (URCA) / Ceara & $1(14,3)$ \\
Universidade Federal de Goiás (UFG) / Goiás & $1(14,3)$ \\
Universidade Federal de Juiz de Fora (UFJF) / Rio de Janeiro & $2(14,3)$ \\
Instituto Federal de Educação, Ciência e Tecnologia de Pernambuco Campus Pesqueira (IFPE)/ & $1(14,3)$ \\
Pernambuco & $\mathrm{N}(\%)$ \\
Titulação do autor & $1(14,3)$ \\
Especialista & $3(42,9)$ \\
Mestrado & $2(28,5)$ \\
Doutorado & $1(14,3)$ \\
\hline Autor se encontra na pesquisa, assistência ou docência & $\mathrm{N}(\%)$ \\
Docência e Pesquisa & $4(57,1)$ \\
Assistência & $2(28,6)$ \\
Docência & $1(14,3)$ \\
\hline
\end{tabular}

Ante o exposto, aponta-se que três (42,9\%) ECR foram oriundos da região Sudeste, três (42,9\%) da região Nordeste e um (14,2\%) da região Centro-oeste.

\section{Discussão}

A escassa e insuficiente quantidade de ensaios clínicos com temáticas referentes à emergência, cadastrados na ReBEC por enfermeiros, mostra-se como achado preocupante. A existência de produção científica de elevado nível de evidência é relevante para subsidiar a divulgação desta temática e o respaldo científico para as atividades gerenciais, administrativas, assistenciais, inerentes ao exercício profissional da enfermagem. Cabe apontar que tais estudos podem impactar na redução da mortalidade e melhoria na qualidade de vida ${ }^{6}$

Quanto ao público pesquisado, observou-se predominância de estudos que investigaram enfermeiros e estudantes de enfermagem. A população leiga como amostra de pesquisa foi encontrada em 
apenas um estudo, o que aponta a ausência de respaldo científico para prática profissional acerca da urgência e emergência no contexto social.

A existência, ainda que tímida e insuficiente, de estudos realizados com estudantes de enfermagem na área de urgência e emergência, aponta preocupação dos pesquisadores que os desenvolveram, pela busca de respaldo científico não somente para a assistência direta em saúde, mas para o ensino acerca das temáticas inerentes ao paciente crítico. Tal fato é relevante pela necessidade social de boa formação na enfermagem, mediante a promoção de um ensino significativo, que consiste na base para o pensamento crítico e tomada de decisão, que contribui para melhor translação do conhecimento para solucionar questões de maneira efetiva ${ }^{7-8}$.

Dentre as variáveis investigadas nos ensaios clínicos, destacou-se a temática acerca de agravos cardiovasculares. No Brasil o número de pessoas acometidas por doenças cardiovasculares torna-se cada vez maior, o que aponta relevância de ensaios clínicos que contemplem o tema e testem intervenções variadas que objetivem o enfrentamento da problemática.

No tocante à intervenção realizada, encontraram-se as medidas de aprendizagem acadêmica com simulação clínica sobre ressuscitação cardiopulmonar. As relevâncias de tais abordagens são corroboradas pesquisas brasileiras, cujos resultados apontam escassez de estudos referentes ao cenário didático/pedagógico e destaca a relevância desses estudos e a necessidade de ampliação, para que surja o equilíbrio em sua aplicação na prática da enfermagem ${ }^{9}$.

Acerca do tipo de mascaramento, apenas um ECR, entre os que constituíram a amostra, teve mascaramento duplo-cego. Sabe-se que a redução de viés na pesquisa científica é fundamental para o alcance de resultados fidedignos; um estudo realizado sobre a resposta inicial de desenvolvimento de ensaios clínicos para Covid-19 apontou que a ausência de mascaramento prejudica os desfechos, com a possibilidade de falhas por violação da randomização podendo inserir vieses ${ }^{10}$.

Entre os tipos de alocação, a maior parte dos estudos tratou-se de ensaios randomizados. A importância da randomização decorre do fato de constituir uma característica que permite, mediante distribuição aleatória dos participantes, aumentar a chance de homogeneidade dos grupos estudados, o que reduz viés aos resultados ${ }^{1}$.

No tocante à filiação dos autores, observou-se que em todos os estudos os autores eram filiados a instituições públicas Federais e Estaduais, o que marca a importância das universidades e institutos públicos para produção científica. Tal resultado converge com estudo sobre grupos de pesquisa em 
enfermagem no Brasil, no qual observa-se predominância das instituições públicas de ensino superior $^{11}$.

A maioria das publicações foram desenvolvidas nas regiões Sudeste e Nordeste. 0 que corrobora com estudo bibliométrico que analisou a produção científica sobre parada cardiorrespiratória nos periódicos brasileiros de enfermagem, onde o resultado apontou que o maior número de estudos sobre PCR foi oriundo da região Sudeste ${ }^{11}$. Tal fato se dá pela centralização de universidades com elevada quantidade de pesquisadores em tal região.

Este estudo apresenta como limitação a probabilidade de perda de Ensaios Clínicos Randomizados selecionáveis para compor a amostra, por não estarem disponíveis no período de coleta de dados no website da ReBEC, este fato pode ser relacionado ao intervalo de tempo entre a aprovação e disponibilidade online.

\section{Conclusão}

Apenas sete estudos sobre urgência e emergência, realizados por enfermeiros cadastrados na ReBEC foram encontrados e não se observou avanço quantitativo ao longo dos anos. Houve predominância de pesquisas oriundas da região sudeste, realizadas por profissionais com titulação de mestre, que atuavam na docência e pesquisa, vinculados a instituições públicas de caráter Federal ou Estadual. Os estudos, predominantemente, investigaram enfermeiros e estudantes, testaram a intervenção de simulação realística, na temática ressuscitação cardiopulmonar, com amostra inferior a 50 participantes. 
Contribuição dos autores

Ana Clara Alves de Brito, Raquel Gouveia Ramos, Girleide Isídio da Silva, Ana Karolina Félix de Almeida, Guilherme Guarino de Moura Sá e Nélson Miguel Galindo Neto participaram na concepção e delineamento do trabalho, na obtenção, análise e interpretação dos dados; na discussão dos resultados, na redação do manuscrito e revisão crítica do seu conteúdo e na aprovação da versão final do manuscrito.

Agradecimentos

Instituto Federal de Pernambuco - Campus Pesqueira e Campus Belo Jardim

Direitos autorais

Este artigo está licenciado sob a Licença Internacional Creative Commons 4.0, tipo BY-NC (https://creativecommons.org/licenses/by-nc/4.0/deed.pt_BR).

\section{(i) (8)}

\section{Referências}

1. Martins WR. Eficácia e Efetividade de Ensaios Clínicos Randomizados: quais as diferenças entre eles? Arq. Bras. Ed. Fís [Internet]. 2020 [citado 03o de maio de 2021];3,(1):09-14. Disponível em: http://dx.doi.org/10.20873/abef.2595-0096.v2n2p914.2020

2. Pacheco RL, Latorraca COC, Oliveira RÁ, Carmo VS, Riera R. Iniciativa COMET. Escolhendo desfechos em ensaios clínicos randomizados. Diagn Tratamento [Internet]. 2019 [citado 03o de maio de 2021];24(1):12-5. Disponível em: https://docs.bvsalud.org/biblioref/2019/06/1005090/rdt-v24n1_12-15.pdf

3. Silva DVA, Oliveira CA, Silva PO, et al. Estratégia para Intensificar a Prática Baseada em Evidência na Formação de Acadêmicos de Enfermagem: Relato de Experiência. Revista de Enfermagem do Centro-Oeste Mineiro [Internet]. 2018 [citado 03ํ de maio de 2021];8:e1784. Disponível em: http://dx.doi.org/10.19175/recom.v9i0.1784

4. Santana FL, Paris MC, Gabriel KOF, Rosa WF, Petry IL, Alves JNB, et al. Atuação do enfermeiro na urgência e emergência: revisão integrativa da literatura. Brazilian Journal of Development [Internet]. 2021 [citado 03ํ de maio de 2021];7(4):35994-35006. Disponível em: https://doi.org/10.34117/bjdv7n4-184

5. Santos MAB, Sá GGM, Caetano JA, Costa AB, Muniz MLC, Galindo Neto NM. Dissertações e teses da enfermagem brasileira acerca da unidade de terapia intensiva. Rev Rene [Internet]. 2017 [citado 03o de maio de 2021];18(4):521-7. Disponível em: http://periodicos.ufc.br/rene/article/view/20256

6. Sorte EMSB et al. Análise da Percepção de Acadêmicos sobre o Ensino de Urgência e Emergência em Curso Médico. Revista Brasileira de Educação Médica [online]. 2020 [citado 01ํo de junho de 2021];44, (03)e075. Disponível em: https://doi.org/10.1590/1981-5271v44.3-20190193

7. Carbogim FC, Luiz FS, Oliveira LB, Braz PR, Santos KB, Püschel VAA. Efetividade de modelo de ensino em um curso de primeiros socorros: ensaio clínico randomizado. Texto Contexto Enferm [Internet]. 2020 [citado $01^{\mathrm{o}}$ de junho de 2021];29:e20180362. Disponível em: http://dx.doi.org/10.1590/1980-265X-TCE-2018-0362

8. Pedrosa KKA, Oliveira ICM, Feijão AR, Machado RC. Enfermagem baseada em evidência: caracterização dos estudos no Brasil. Cogitare Enferm. [Internet]. 2015 [citado 01ํo de junho de 2021];20(4): 733-741. Disponível em: https://docs.bvsalud.org/biblioref/2016/08/1128/40768-166899-1-pb.pdf

9. Ribeiro TB et al. Avaliação da resposta inicial de desenvolvimento de ensaios clínicos para COVID-19 no Brasil. Revista Brasileira de Epidemiologia [online]. 2020 [citado 16o de agosto de 2021];23 e200104. Disponível em: https://doi.org/10.1590/1980-549720200104

10. Erdmann AL, Peiter CC, Lanzoni, GMM. Grupos de pesquisa em enfermagem no Brasil: comparação dos perfis de 2006 e 2016. Rev Gaúcha Enferm [Internet]. 2017 [citado 16o de agosto de 2021];38(2):e69051. Disponível em: https://doi.org/10.1590/1983-1447.2017.02.69051

11. Galindo Neto NM, Martins KUSC, Silva PMN, Silva RX, Alexandre ACS, Sá GGM. Produção científica sobre parada cardiorrespiratória nos periódicos brasileiros de enfermagem: estudo bibliométrico. Rev baiana enferm [Internet]. (2020) [citado 16º de agosto de 2021];34:e36363. Disponível em:

https://doi.org/10.18471/rbe.v34.36363 


\begin{abstract}
Objective: To characterize the randomized clinical trials on urgency and emergency carried out by nurses in Brazil. Method: Descriptive, quantitative study. Carried out in the laboratory of the Federal Institute of Pernambuco Campus Pesqueira, through access to the online database of the Brazilian Clinical Trials Registry. In data collection, an instrument was applied, consisting of 23 questions that were filled out according to information from each study. For data analysis, the Microsoft Excel 2016 software was used. Results: Of the 321 studies registered at ReBEC, seven (2.1\%) corresponded to surveys carried out on urgency and emergency. As for the assessed public, two studies (28.6\%) investigated nurses, two (28.6\%) were carried out with nursing students, two $(28.6 \%)$ researched patients and one $(14.2 \%)$ was constituted for deaf students. Regarding the type of masking, five (71.4\%) studies were opened; one $(14.3 \%)$ was single-blind and one (14.3\%) was double-blind. All authors were affiliated to public institutions of a Federal or State nature. Conclusion: This study points out the limited role of nursing in conducting Randomized Clinical Trials related to urgency and emergency in Brazil.
\end{abstract}

Descriptors: Randomized Clinical Trials; Nursing; Emergency.

Keywords: Nurses; Clinical trials; Urgency and emergency.

\title{
RESUMEN
}

Objetivo: caracterizar los ensayos clínicos aleatorizados sobre urgencia y emergencia realizados por enfermeras en Brasil. Método: Estudio descriptivo, cuantitativo. Realizado en el laboratorio del Instituto Federal de Pernambuco Campus Pesqueira, mediante acceso a la base de datos en línea del Registro Brasileño de Ensayos Clínicos. En la recolección de datos se aplicó un instrumento compuesto por 23 preguntas que se completaron de acuerdo con la información de cada estudio. Para el análisis de los datos se utilizó el software Microsoft Excel 2016. Resultados: De los 321 estudios registrados en ReBEC, siete (2,1\%) correspondieron a encuestas realizadas sobre urgencia y emergencia. En cuanto al público evaluado, dos estudios $(28,6 \%)$ investigaron enfermeras, dos $(28,6 \%)$ se realizaron con estudiantes de enfermería, dos $(28,6 \%)$ pacientes investigados y uno $(14,2 \%)$ se constituyó para estudiantes sordos. En cuanto al tipo de enmascaramiento, se abrieron cinco (71,4\%) estudios; uno (14,3\%) era simple ciego y uno (14,3\%) era doble ciego. Todos los autores estaban afiliados a instituciones públicas de carácter federal o estatal. Conclusión: Este estudio señala el papel limitado de la enfermería en la realización de Ensayos Clínicos Aleatorizados relacionados con la urgencia y la emergencia en Brasil.

Descriptores: Ensayos Clínicos Aleatorios; Enfermería; Urgencia.

Palabras clave: Enfermeras; Ensayos clínicos; Urgencia y emergencia. 